\title{
Notes on Free Volume Theories
}

\author{
Hiroshi Fujita \\ 35 Shimotakedono-cho, Shichiku, Kita-ku, Kyoto, Japan
}

(Received June 10, 1991)

\begin{abstract}
There are two typical formulations for the mobilities of jumping units in concentrated polymer solutions on the basis of free volume concept. One is due to Fujita and the other to Vrentas and Duda. The prevailing notion that the former is contained as a special case of the latter is not correct, because the two theories are built on significantly different assumptions. The present paper clarifies this point and gives new evidence for the Fujita-Kishimoto approximation to the composition dependence of the fractional free volume.
\end{abstract}

KEY WORDS Free Volume Theory / Cohen-Turnbull Theory / Free Volume / Fractional Free Volume / Mobility / Doolittle Equation / Fujita Theory / Vrentas-Duda Theory / Critical Hole Volume / Hole Volume Assumption / Concentrated Polymer Solutions /

Free volume is a useful concept for discussing the mobilities of the solvent and chain segments in concentrated polymer solutions. Typical of its formulation are the earlier theory due to Fujita ${ }^{1}$ and the subsequent one to Vrentas and Duda. ${ }^{2}$ These two theories are built on different postulates, but it does not seem that this fact is well recognized in general. The present paper aims to clarify important differences of the two theories by summarizing their key assumptions and results. Paul's free volume theory ${ }^{3}$ is not taken up here, because it is essentially a modified version of the Vrentas-Duda theory.

The molecules in liquids are given much greater freedom of motion than those in solids. They can migrate thermally over a distance of macroscopic scale. This feature is attributed to the existence of holes or interstitial space through which each molecule can move rather freely, and a quantity called free volume is introduced as a measure of such space. The frequency of molecular jumping should be larger as there is more chance for the molecule to find a hole in its vicinity. In 1959, calculating this chance on the assumption that redistribu- tion of holes requires no change in energy, Cohen and Turnbull ${ }^{4}$ derived for pure liquids

$$
\ln m=\ln m_{0}-\gamma v_{\mathrm{c}} / v_{\mathrm{f}}
$$

where $m$ is the molecular mobility defined as the velocity with which a molecule translates steadily under the action of unit force (its inverse is the molecular friction coefficient), $m_{0}$ a constant having the dimension of $m, \gamma$ a numerical factor between 0.5 and $1, v_{\mathrm{c}}$ the volume of a minimum hole that has to exist in the neighbor of the molecule concerned (this is hereafter called the critical hole volume), and $v_{\mathrm{f}}$ the free volume per molecule. It seems reasonable equating $v_{\mathrm{c}}$ to the volume occupied by the entity of one molecule. The point is that the Cohen-Turnbull theory does not quantify $v_{\mathrm{f}}$ explicitly in terms of known molecular parameters so that it does not allow us to envisage the physical model of the free volume. Probably, for such reason, the free volume is often interpreted as the space in the liquid not occupied by the cores of the constituent molecules. If this interpretation, which hereafter is called the hole volume assumption, is accepted, $v_{\mathrm{f}}$ can be equated to $v-v_{\mathrm{c}}$, where $v$ 
is the liquid volume per molecule, and eq 1 yields

$$
\ln m=C-\gamma v / v_{\mathrm{f}}
$$

where $C$ is a constant. We introduce a dimensionless quantity $f$ called the fractional free volume of the system and defined by

$$
f=V_{\mathrm{F}} / V
$$

where $V$ is the specific volume of a given liquid and $V_{\mathrm{F}}$ the free volume contained in it, i.e., the specific free volume. For a pure liquid we have $V=N v$ and $V_{\mathrm{F}}=N v_{\mathrm{f}}$, where $N$ is the total number of molecules per gram of liquid, so that $v / v_{\mathrm{f}}=V / V_{\mathrm{F}}=1 / f$, and eq 2 can be written

$$
\ln m=C-\gamma / f
$$

Experimentally, $V$ is measurable, and $N v_{\mathrm{c}}$ may be estimated from appropriate volume data. If the hole volume assumption is accepted, $V_{\mathrm{F}}=V-N v_{\mathrm{c}}$, and hence $V_{\mathrm{F}}$ is calculable. Thus, $f$ becomes a measurable quantity. This means that, with the hole volume assumption, the temperature dependence of $m$ for pure liquids can be predicted from experimental information on $V$ and $v_{\mathrm{c}}$.

In 1957, Doolittle ${ }^{5}$ proposed for the viscosity $\eta$ of pure liquids an empirical equation which can be written in terms of $f$ as

$$
\ln \eta=A^{\prime}+B / f
$$

where $A^{\prime}$ and $B$ and constants. Later, Williams et $a l .{ }^{6}$ showed that eq 5 fits accurately the temperature dependence of $\eta$ for polymeric solids if $f$ is assumed to vary linearly with temperature. Since $\eta$ is inversely proportional to $m$ for pure liquids, the Doolittle equation gives

$$
\ln m=A-B / f
$$

with $A$ being a new constant. The formal agreement of eq 6 with eq 4 is often regarded as giving a theoretical support to Doolittle's proposal. However, it should be noted that eq 6 cannot be derived from eq 1 without invoking the hole volume assumption.

Though appealing to our physical intuition, the hole volume assumption has no a priori justfication. Without this assumption the Doolittle equation bears no relation with the Cohen-Turnbull theory, but it simply correlates $m$ to $f$, the free volume per unit volume of solution. The same can be said of eq 1 , which correlates $m$ to $v_{\mathrm{f}}$, the free volume per jumping molecule. The two correlations are essentially equivalent to each other for pure liquids, but lead to different predictions for solutions. One of the aims of the present paper is to highlight this point.

As mentioned above, the typical free volume formulations of polymer solutions are the theory of Vrentas and Duda ${ }^{2}$ and the theory of Fujita. ${ }^{1}$ In either theory, it is assumed that the jumping unit is a single molecule for the solvent component and a chain segment for the polymer component. Vrentas and Duda postulate that the mobility of either jumping unit is governed by the average free volume per jumping unit through the Cohen-Turnbull equation 1 and calculate this average by using the hole volume assumption. On the other hand, Fujita correlates the mobility of either jumping unit to the free volume per unit volume of solution through the Doolittle equation 6 .

The Vrentas-Duda theory is capable of predicting the composition dependence of the mobilities of the jumping units with the parameters which all can in principle be estimated with a set of data on pure components. Thus it has a predictive capability and is quite inviting for practical work. On the other hand, the Fujita theory has no such power, but focuses on determining the temperature and composition dependence of $f$ in actual polymers solutions from appropriate mobility data. It is shown below that the information of $f$ so obtained allows us to evalaute the fractional free volume associated with each jumping unit. 


\section{THE VRENTAS-DUDA THEORY ${ }^{2}$}

\section{Basic Assumptions and Equations}

First, we outline the Vrentas-Duda theory, with the subscripts $\mathrm{s}$ and $\mathrm{p}$ used to denote the quantities associated with the solvent and the polymer, respectively, and the subscript pj to designate the quantities related to the polymer jumping unit, i.e., a chain segment. If the hole volume assumption is accepted, the specific free volume of the solution, $V_{\mathrm{F}}$, is expressed by

$$
V_{\mathrm{F}}=V-V_{\mathrm{M}}
$$

where, as defined earlier, $V$ is the specific volume of the solution, and $V_{\mathrm{M}}$ represents the volume occupied by the cores of all solvent and polymer molecules in one gram of the solution. The total number of jumping units, $N$, contained in one gram of the solution is given by

$$
N=N_{\mathrm{A}}\left(w_{\mathrm{s}} / M_{\mathrm{s}}+w_{\mathrm{pj}} / M_{\mathrm{pj}}\right)
$$

where $N_{\mathrm{A}}$ is the Avogadro constant, $w_{\mathrm{s}}$ and $M_{\mathrm{s}}$ the weight fraction and molecular weight of the solvent, and $w_{\mathrm{pj}}$ and $M_{\mathrm{pj}}$ the corresponding quantities for the chain segment. On average, a free volume equal to $V_{\mathrm{F}} / N$ is shared to each jumping unit. Vrentas and Duda used eq 1 to express the mobility $m_{\mathrm{s}}$ of the solvent and the mobility $m_{\mathrm{pj}}$ of the chain segment by substituting $V_{\mathrm{F}} / N$ for $v_{\mathrm{f}}$, with $v_{\mathrm{c}}$ replaced by $V_{\mathrm{s}} M_{\mathrm{s}} / N_{\mathrm{A}}$ for the solvent and by $V_{\mathrm{pj}} M_{\mathrm{pj}} / N_{\mathrm{A}}$ for the chain segment. Here, $V_{\mathrm{x}}$ denotes the specific critical hole volume of a jumping unit $\mathrm{x}$, so that $V_{\mathrm{x}} M_{\mathrm{x}} / N_{\mathrm{A}}$ stands for the critical hole volume of that unit.

This Vrentas-Duda extension of the CohenTurnbull theory gives for $m_{\mathrm{s}}$

$$
\ln m_{\mathrm{s}}=\ln m_{\mathrm{s} 0}-\gamma V_{\mathrm{s}} M_{\mathrm{s}} N /\left(N_{\mathrm{A}} V_{\mathrm{F}}\right)
$$

which, on substitution of eq 8 , yields

$$
m_{\mathrm{s}}=m_{\mathrm{s} 0} \exp \left[-\gamma\left(w_{\mathrm{s}} V_{\mathrm{s}}+w_{\mathrm{pj}} \xi V_{\mathrm{pj}}\right) / V_{\mathrm{F}}\right]
$$

where $\xi$ is a dimensionless quantity defined by

$$
\xi=\frac{M_{s} V_{\mathrm{s}}}{M_{\mathrm{pj}} V_{\mathrm{pj}}}
$$

and denotes the molar critical hole volume of the solvent relative to that of the chain segment. The corresponding expression for $m_{\mathbf{p j}}$ is

$m_{\mathrm{pj}}=m_{\mathrm{pj} 0} \exp \left[-\gamma\left(w_{\mathrm{s}} V_{\mathrm{s}}+w_{\mathrm{p}} \xi V_{\mathrm{pj}}\right) /\left(\xi V_{\mathrm{F}}\right)\right]$

Vrentas and Duda express $V_{\mathrm{M}}$ as

$$
\begin{aligned}
V_{\mathrm{M}}= & w_{\mathrm{s}} V_{\mathrm{Ms}}(0) \exp \left(\int_{0}^{T} \alpha_{\mathrm{cs}} \mathrm{d} T\right) \\
& +w_{\mathrm{p}} V_{\mathrm{Mp}}(0) \exp \left(\int_{0}^{T} \alpha_{\mathrm{cp}} \mathrm{d} T\right)
\end{aligned}
$$

where $T$ is the absolute temperature, $V_{\mathbf{M s}}(0)$ and $V_{\mathrm{Mp}}(0)$ the specific core volumes of the solvent and polymer in the pure state at $T=0$, respectively, and $\alpha_{c s}$ and $\alpha_{c p}$ the thermal expansivites of these volumes. With eq 13 , eq 7 can be transformed to

$$
V_{\mathrm{F}}=w_{\mathrm{s}} V_{\mathrm{F}}(1)+w_{\mathrm{p}} V_{\mathrm{F}}(0)+\Delta V\left(w_{\mathrm{s}}\right)
$$

where $V_{\mathrm{F}}(1)$ and $V_{\mathrm{F}}(0)$ are the values of $V_{\mathrm{F}}\left(w_{\mathrm{s}}\right)$ at $w_{\mathrm{s}}=1$ and 0 , respectively, and $\Delta V\left(w_{\mathrm{s}}\right)$ is defined by

$$
\Delta V\left(w_{\mathrm{s}}\right)=V-w_{\mathrm{s}} V(\mathrm{~s})-w_{\mathrm{p}} V(\mathrm{p})
$$

with $V(\mathrm{~s})$ and $V(\mathrm{p})$ denoting the specific volumes of the pure solvent and polymer, respectively. Thus we see that $\Delta V\left(w_{\mathrm{s}}\right)$ represents the change in $V$ on mixing. In general, this change may be neglected in a good approximation. Then, eq 10 and eq 12 reduce to

$$
\begin{aligned}
m_{\mathrm{s}}= & m_{\mathrm{s} 0} \exp \left\{-\gamma\left(w_{\mathrm{s}} V_{\mathrm{s}}\right.\right. \\
& \left.\left.+w_{p} \xi V_{\mathrm{pj}}\right) /\left[w_{s} V_{\mathrm{F}}(1)+w_{p} V_{\mathrm{F}}(0)\right]\right\} \\
m_{\mathrm{pj}}= & m_{\mathrm{pj} 0} \exp \left\{-\gamma\left(w_{s} V_{\mathrm{s}}\right.\right. \\
& \left.\left.+\omega_{\mathrm{p}} \xi V_{\mathrm{pj}}\right) /\left[\xi\left(w_{\mathrm{s}} V_{F}(1)+w_{p} V_{F}(0)\right)\right]\right\}
\end{aligned}
$$

For $\Delta V=0$ the following relations hold between $w_{\mathrm{s}}$ and the solvent volume fraction $\phi_{\mathrm{s}}$ and between $w_{\mathrm{p}}$ and the polymer volume fraction $\phi_{\mathrm{p}}$ :

$$
\begin{gathered}
V(\mathrm{~s}) w_{\mathrm{s}}=V \phi_{\mathrm{s}} \\
V(\mathrm{p}) w_{\mathrm{p}}=V \phi_{\mathrm{p}}
\end{gathered}
$$


which allow eq 16 and eq 17 to be rewritten

$$
\begin{aligned}
m_{\mathrm{s}}= & m_{\mathrm{s} 0} \exp \left[-\gamma\left(\phi_{\mathrm{s}} \bar{V}_{\mathrm{s}}+\phi_{\mathrm{p}} \xi \bar{V}_{\mathrm{pj}}\right) /\left(\phi_{\mathrm{s}} f_{\mathrm{s}}+\phi_{\mathrm{p}} f_{\mathrm{p}}\right)\right] \\
m_{\mathrm{pj}}= & m_{\mathrm{pj} 0} \exp \left\{-\gamma\left(\phi_{\mathrm{s}} \bar{V}_{\mathrm{s}}\right.\right. \\
& \left.\left.+\phi_{\mathrm{p}} \xi \bar{V}_{\mathrm{pj}}\right) /\left[\xi\left(\phi_{\mathrm{s}} f_{\mathrm{s}}+\phi_{\mathrm{p}} f_{\mathrm{p}}\right)\right]\right\}
\end{aligned}
$$

where

$$
\begin{gathered}
\bar{V}_{\mathrm{s}}=V_{\mathrm{s}} / V(\mathrm{~s}) \\
\bar{V}_{\mathrm{pj}}=V_{\mathrm{pj}} / V(\mathrm{p}) \\
f_{\mathrm{s}}=V_{\mathrm{F}}(1) / V(\mathrm{~s}) \\
f_{\mathrm{p}}=V_{\mathrm{F}}(0) / V(\mathrm{p})
\end{gathered}
$$

The quantities $f_{\mathrm{s}}$ and $f_{\mathrm{p}}$ represent the fractional free volume in the pure solvent and in the pure polymer, respectively. According to eq 20 and eq 21, the composition dependence of $m_{\mathrm{s}}$ and $m_{\mathrm{pj}}$ is governed by five parameters $\xi, \bar{V}_{\mathrm{s}}, \bar{V}_{\mathrm{pj}}$, $f_{\mathrm{s}}$, and $f_{\mathrm{p}}$, and these equations show that $\gamma \bar{V}_{\mathrm{s}} / f_{\mathrm{s}}$, $\gamma \bar{V}_{\mathrm{pj}} / f_{\mathrm{p}}$, and $\xi$ can in principle be evaluated from temperature dependence data of $m_{\mathrm{s}}$ at $\phi_{\mathrm{s}}=0$ and 1 as well as that of $m_{\mathrm{pj}}$ at $\phi_{\mathrm{s}}=0$ if, as usually the case, those data follow the WLF type of equation. ${ }^{6}$ To make the determination of all five paramenters possible Vrentas and Duda introduced an assumption that they claimed to be reasonable. It is to equate $V_{s}$ and $V_{\mathrm{pj}}$ to $V_{\mathrm{Ms}}(0)$ and $V_{\mathrm{Mp}}(0)$, respectively. According to them, the latter can be estimated by method of Haward. ${ }^{7}$ Both $V(\mathrm{~s})$ and $V(\mathrm{p})$ are directly measurable. Hence, it is possible to determine $\bar{V}_{\mathrm{s}}$ and $\bar{V}_{\mathrm{pj}}$ and, in turn, $f_{\mathrm{s}} / \gamma$ and $f_{\mathrm{p}} / \gamma$. Summarizing, we can evaluate all the five parameters mentioned above, so that the Vrentas-Duda theory is capable of predicting the composition dependence of both $m_{\mathrm{s}}$ and $m_{\mathrm{pj}}$ for a polymer solution with experimental information on the pure components. This is one of the most remarkable features of the Vrentas-Duda free volume formulation.

\section{Comments}

On the Postulate. According to the postulate made by Vrentas and Duda, $m_{\mathrm{s}}$ is described by eq 9. This equation indicates that for given $V_{F}$ the value of $m_{\mathrm{s}}$ varies with $N$, which, as can be seen from eq 8 , depends on $M_{\mathrm{pj}}$ for given $w_{\mathrm{s}}$ and $M_{\mathrm{s}}$. Hence, for a polymer solution which has specified $V_{\mathrm{F}}$ and $w_{\mathrm{s}}, m_{\mathrm{s}}$ depends not only on $M_{\mathrm{s}}$ but also on $M_{\mathrm{pj}}$, i.e., on the size of the chain segment. In fact, as this size is chosen smaller, $N$ becomes larger and hence $m_{\mathrm{s}}$ smaller, i.e., the solvent gets more difficult to move. Such a consequence seems irrational.

Relation with the Fujita-Kishimoto Theory. Verntas and Duda ${ }^{2}$ derived from eq 16

$$
\ln \frac{m_{\mathrm{s}}\left(\phi_{\mathrm{s}}\right)}{m_{\mathrm{s}}(0)}=\frac{\bar{B} \bar{\beta} V \phi_{\mathrm{s}}}{V(\mathrm{p}) f_{\mathrm{p}}^{2}+\bar{\beta} f_{\mathrm{p}} V \phi_{\mathrm{s}}}
$$

where

$$
\begin{gathered}
\bar{B} \bar{\beta}=\gamma\left[\left(\xi V_{\mathbf{p} \mathbf{j}} / V(\mathrm{p})\right) f_{\mathrm{s}}-\left(V_{\mathrm{s}} / V(\mathrm{~s})\right) f_{\mathrm{p}}\right] \\
\bar{\beta}=f_{\mathrm{s}}-(V(\mathrm{p}) / V(\mathrm{~s})) f_{\mathrm{p}}
\end{gathered}
$$

Here, the temperature dependence of respective quantities is not indicated explicitly. This convention will be used throughout the subsequent presentation. Since for $\Delta V=0$ we have

$$
V / V(\mathrm{p})=1+[V(\mathrm{~s})-V(\mathrm{p})] w_{\mathrm{s}} / V(\mathrm{p})
$$

Vrentas and Duda considered that $V$ in eq 26 may be replaced by $V(\mathrm{p})$ under the condition

$$
[V(\mathrm{~s})-V(\mathrm{p})] w_{\mathrm{s}} / V(\mathrm{p}) \ll 1
$$

which holds for very concentrated solutions near the pure polymer. With this substitution, eq 26 reduces to

$$
\ln \frac{m_{\mathrm{s}}\left(\phi_{\mathrm{s}}\right)}{m_{\mathrm{s}}(0)}=\frac{\bar{B} \bar{\beta} \phi_{\mathrm{s}}}{f_{\mathrm{p}}^{2}+\bar{\beta} f_{\mathrm{p}} \phi_{\mathrm{s}}}
$$

which agrees with

$$
\ln \frac{m_{\mathrm{s}}\left(\phi_{\mathrm{s}}\right)}{m_{\mathrm{s}}(0)}=\frac{B \beta \phi_{\mathrm{s}}}{f_{\mathrm{p}}^{2}+\beta f_{\mathrm{p}} \phi_{\mathrm{s}}}
$$

that Fujita and Kishimoto ${ }^{8}$ derived from their free volume formulation described below, through $B \beta$ and $\beta$ differ from $\bar{B} \bar{\beta}$ and $\bar{\beta}$ in the contents. From this formal agreement Vrentas and Duda concluded that the Fujita-Kishimoto theory is a special case of their more general 
theory valid under the condition specified by eq 30 . However, we note that eq 26 can be transformed into an expression conforming to eq 32 with $\beta=f_{\mathrm{s}}-f_{\mathrm{p}}$ without invoking eq 30 ; the derivation is made by using the relation

$$
1 / V=(1 / V(\mathrm{p}))\left\{1-\left[1-(V(\mathrm{p}) / V(\mathrm{~s})) \phi_{\mathrm{s}}\right]\right\}
$$

which follows from eq 18 and 29 . Thus, the conclusion of Vrentas and Duda is not correct.

\section{THE FUJITA THEORY ${ }^{1}$}

\section{Basic Assumptions and Equations}

Fujita postulated that $m_{\mathrm{s}}$ and $m_{\mathrm{pj}}$ are controlled by the fractional free volume $f$ of the solution through a set of the Doolittle equations:

$$
\begin{gathered}
\ln m_{\mathrm{s}}=A_{\mathrm{s}}-B_{\mathrm{s}} / f \\
\ln m_{\mathrm{pj}}=A_{\mathrm{pj}}-B_{\mathrm{pj}} / f
\end{gathered}
$$

where the parameters $A_{\mathrm{s}}$, etc. are assumed to be independent of external variables such as temperature and concentration. Either eq 34 or eq 35 should be taken as the defining expression for $f$. But if the latter is chosen as such, $B_{\mathbf{p j}}$ may be absorbed into $f$ without loss of generality. Thus we may redefine $f$ by

$$
\ln m_{\mathrm{pj}}=A_{\mathrm{pj}}-1 / f
$$

Then, we must replace eq 34 by

$$
\ln m_{\mathrm{s}}=A_{\mathrm{s}}-B / f
$$

where $B$ stands for $B_{\mathrm{s}} / B_{\mathrm{pj}}$. Obviously, it is meaningless to speak of the absolute value of the redefined $f$. Frisch et al. ${ }^{9}$ proposed using different $f$ for $m_{\mathrm{s}}$ and $m_{\mathrm{pj}}$, but the Fujita theory prefers simplicity by assigning the same $f$ to both jumping units. As mentioned above, the Ventas-Duda theory assumes the free volume per jumping unit as the controlling factor for $m_{\mathrm{s}}$ and $m_{\mathrm{pj}}$. On the other hand, the Fujita theory assumes the fractional free volume of the solution, i.e., the free volume per unit volume of solution, as such a factor. We again emphasize that clear recognition of this difference is crucial for understanding the two theories.

The composition dependence of $m_{\mathrm{pj}}$ can be obtained from measurements of polymer self-diffusion coefficient or solution viscosity at a series of concentrations. Then, that of $m_{\mathrm{s}}$ can be calculated by use of the relation

$$
m_{\mathrm{s}} \sim\left(m_{\mathrm{pj}}\right)^{\boldsymbol{B}}
$$

which follows from eq 36 and 37, provided that $B$ is known. Experimental evidence for eq 38 was presented long ago ${ }^{1}$ for several systems at the limit $\phi_{\mathrm{s}} \rightarrow 0$, and it was found that $B$ is smaller than unity for all the systems examined. However, as will be noted below, $B$ must be unity at the limit $\phi_{\mathrm{s}} \rightarrow 1$.

It can be shown that the Vrentas-Duda theory predicts $m_{\mathrm{s}} \sim\left(m_{\mathrm{pj}}\right)^{\xi}$. Thus, $B$ in the Fujita theory may be interpreted as having the physical meaning of $\xi$, i.e., the molar critical volume of the solvent relative to that of the chain segment, but the fact that $B \rightarrow 1$ as $\phi_{\mathrm{s}} \rightarrow 1$ suggests the breakdown of this interpretation at a certain composition.

\section{Fujita-Kishimoto Approximation}

The free volume is an extensive quantity. Therefore, if no volume change occurs on mixing, it can be shown by the familiar method in solution thermodynamics that $f\left(\phi_{\mathrm{s}}\right)$ is represented by

$$
f\left(\phi_{\mathrm{s}}\right)=\bar{f}_{\mathrm{s}}\left(\phi_{\mathrm{s}}\right) \phi_{\mathrm{s}}+\bar{f}_{\mathrm{p}}\left(\phi_{\mathrm{s}}\right) \phi_{\mathrm{p}}
$$

with the intensive properties $\bar{f}_{\mathrm{s}}$ and $\bar{f}_{\mathrm{p}}$ defined by

$$
\begin{aligned}
& \bar{f}_{\mathrm{s}}\left(\phi_{\mathrm{s}}\right)=\left(\partial \hat{V}_{\mathrm{F}} / \partial \hat{V}_{\mathrm{s}}\right)_{T} \\
& \bar{f}_{\mathrm{p}}\left(\phi_{\mathrm{s}}\right)=\left(\partial \hat{V}_{\mathrm{F}} / \partial \hat{V}_{\mathrm{p}}\right)_{T}
\end{aligned}
$$

Here, $\hat{V}_{F}$ is the free volume contained -in a solution, and $\hat{V}_{\mathrm{s}}$ and $\hat{V}_{\mathrm{p}}$ are the volumes of the solvent and polymer in that solution, respectively. The quantity $\bar{f}_{\mathrm{s}}$ (or $\bar{f}_{\mathrm{p}}$ ) represents the change in $\hat{V}_{\mathrm{F}}$ that occurs when a unit volume of solvent (or of polymer) is added to the solution, with $\hat{V}_{\mathrm{p}}$ (or $\hat{V}_{\mathrm{s}}$ ) held constant. Hence, 
$\bar{f}_{\mathrm{s}}$ and $\bar{f}_{\mathrm{p}}$ may be interpreted, respectively, as the fractional free volumes associated with unit volume of solvent and unit volume of polymer in a given solution.

If $\bar{f}_{\mathrm{s}}$ and $\bar{f}_{\mathrm{p}}$ do not depend on composition, they are equal to $\bar{f}_{\mathrm{s}}(1)$ and $\bar{f}_{\mathrm{p}}(0)$, respectively. In this case, eq 39 yields

$$
f\left(\phi_{\mathrm{s}}\right)=\bar{f}_{\mathrm{p}}^{0}+\left(\bar{f}_{\mathrm{s}}^{0}-\bar{f}_{\mathrm{p}}^{0}\right) \phi_{\mathrm{s}}
$$

where the superscript 0 signifies the pure state of each component. This equation is equivalent to what was assumed by Kelley and Bueche ${ }^{10}$ in 1961. In the same year, Fujita and Kishimoto ${ }^{8}$ proposed

$$
f\left(\phi_{\mathrm{s}}\right)=\bar{f}_{\mathrm{p}}^{0}+\beta \phi_{\mathrm{s}}
$$

with

$$
\beta=C-\bar{f}_{\mathbf{p}}^{0}
$$

Here, $C$ is an adjustable function of temperature, but the formulation of Fujita and Kishimoto indicates that it is actually the value of $\bar{f}_{\mathrm{s}}$ at the limit $\phi_{\mathrm{s}} \rightarrow 0$, i.e., in the solution very close to the pure polymer. With this limiting value designated by $\bar{f}_{\mathrm{s}}^{*}$, eq 43 is written

$$
f\left(\phi_{\mathrm{s}}\right)=\bar{f}_{\mathrm{p}}^{0}+\left(\bar{f}_{\mathrm{s}}^{*}-\bar{f}_{\mathrm{p}}^{0}\right) \phi_{\mathrm{s}}
$$

Combining eq 43 with eq 37 yields

$$
\ln \frac{m_{\mathrm{s}}\left(\phi_{\mathrm{s}}\right)}{m_{\mathrm{s}}(0)}=\frac{B \beta \phi_{\mathrm{s}}}{\left(\bar{f}_{\mathrm{p}}^{0}\right)^{2}+\beta \bar{f}_{\mathrm{p}}^{0} \phi_{\mathrm{s}}}
$$

which is nothing but eq 32 .

The Fujita-Kishimoto approximation corresponds to the special case of eq 39 in which $\bar{f}_{\mathrm{s}}\left(\phi_{\mathrm{s}}\right)$ and $\bar{f}_{\mathrm{p}}\left(\phi_{\mathrm{s}}\right)$ are replaced by their values at the limit $\phi_{\mathrm{s}} \rightarrow 0$. Therefore, it should be adequate only for very concentrated solutions. However, as is often reported, ${ }^{11}$ it applies up to larger $\phi_{\mathrm{s}}$ than originally expected. The answer to why this was the case is obtained if $f\left(\phi_{\mathrm{s}}\right)$ is evaluated experimentally over a wide range of $\phi_{\mathrm{s}}$, because it is then possible to calculate $\bar{f}_{\mathrm{s}}$ and $\bar{f}_{\mathbf{p}}$ from

$$
\bar{f}_{\mathrm{s}}\left(\phi_{\mathrm{s}}\right)=f\left(\phi_{\mathrm{s}}\right)+\left(1-\phi_{\mathrm{s}}\right)\left(\partial f / \partial \phi_{\mathrm{s}}\right)_{T}
$$

$$
\bar{f}_{\mathrm{p}}\left(\phi_{\mathrm{s}}\right)=f\left(\phi_{\mathrm{s}}\right)-\phi_{\mathrm{s}}\left(\partial f / \partial \phi_{\mathrm{s}}\right)_{T}
$$

which can be derived by noticing that $\bar{f}_{\mathrm{s}}$ and $\bar{f}_{\mathrm{p}}$ satisfy a relation similar to the Gibbs-Duhem relation in solution thermodynamics.

As is well known, the steady viscosity $\eta$ of entangled polymer solutios obeys an empirical relation $^{6}$

$$
\eta \sim\left(1-\phi_{\mathrm{s}}\right)^{3.5} M_{w}^{3.5} / m_{\mathrm{pj}}
$$

where $M_{w}$ is the weight-average molecular weight of the polymer (the small difference of the exponent 3.5 from the familiar 3.4 does not matter here). Denoting the value of $\eta$ for the pure polymer by $\eta_{\mathrm{p}}^{0}$ and defining $Z_{\mathrm{pj}}\left(\phi_{\mathrm{s}}\right)$ by

$$
Z_{\mathrm{pj}}\left(\phi_{\mathrm{s}}\right)=\ln \left[m_{\mathrm{pj}}\left(\phi_{\mathrm{s}}\right) / m_{p j}(0)\right]
$$

we can derive from eq 36 and 49

$$
Z_{\mathrm{pj}}=\ln \left[\left(1-\phi_{\mathrm{s}}\right)^{3.5} \eta_{\mathrm{p}}^{0} / \eta\left(\phi_{\mathrm{s}}\right)\right]=1 / \bar{f}_{\mathrm{p}}^{0}-1 / f\left(\phi_{\mathrm{s}}\right)
$$

and

$$
\eta_{\mathbf{p}}^{0} \sim \exp \left(1 / \bar{f}_{\mathbf{p}}^{0}\right)
$$

If, as is usually the case, the temperature dependence of $\eta_{\mathrm{p}}^{0}$ follows the WLF type of equation, eq 52 allows $\bar{f}_{\mathrm{p}}^{0}$ to be determined experimentally. Then, $f\left(\phi_{\mathrm{s}}\right)$ can be computed from eq 51, because $Z_{\mathrm{pj}}\left(\phi_{\mathrm{s}}\right)$ is calculable from $\eta\left(\phi_{\mathrm{s}}\right)$ data.

Fujita and Einaga ${ }^{12}$ applied this method to published viscosity data on some typical polymer + solvent systems. Typical results are illustrated in Figures 1 and 2, which show features that all the systems examined have in common. In the figures, the arrows indicate what is called the overlap concentration. Substantial chain entanglement would occur at polymer concentrations much higher than this. For either system $f\left(\phi_{\mathrm{s}}\right)$ keeps increasing linearly as $\phi_{\mathrm{p}}$ decreases from unity to a fairly small value. If the slope $\partial f / \partial \phi_{\mathrm{p}}$ of this linear part is denoted by $-\varepsilon$, it follows from eq 39 that $\varepsilon=\bar{f}_{\mathrm{s}}^{*}-\bar{f}_{\mathrm{p}}^{0}$, where $\bar{f}_{\mathrm{s}}^{*}$ has been defined above, and eq 47 and 48 give $\bar{f}_{\mathrm{s}}=\bar{f}_{\mathrm{s}}^{*}$ and $\bar{f}_{\mathrm{p}}=\bar{f}_{\mathrm{p}}^{0}$, respectively. This means that $\bar{f}_{\mathrm{s}}$ and $\bar{f}_{\mathrm{p}}$ remain 


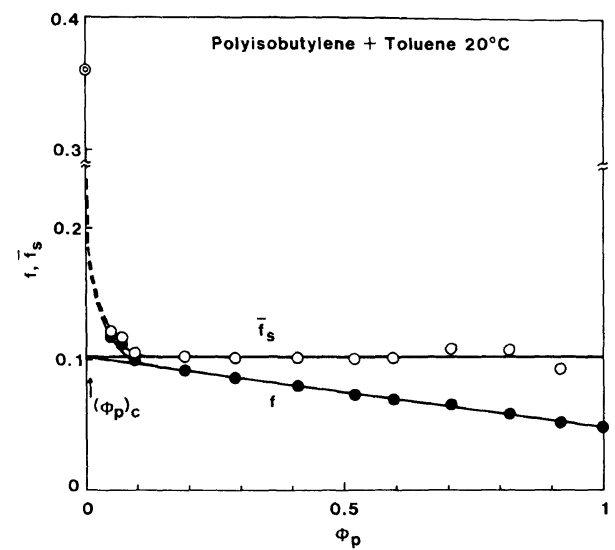

Figure 1. Composition dependence of fractional free volume $f\left(\phi_{\mathrm{s}}\right)(\mathbf{O})$ and solvent fractional free volume $\overline{\mathrm{f}}_{\mathrm{s}}\left(\phi_{\mathrm{s}}\right)$ (O) for polyisobutylene in cyclohexane at $20^{\circ} \mathrm{C} . \phi_{\mathrm{s}}$ and $\phi_{\mathrm{p}}\left(=1-\phi_{\mathrm{s}}\right)$ are the volume fractions of solvent and polymer, respectively. The unfilled circles were obtained from the filled ones with eq 39 and $\bar{f}_{\mathbf{p}}\left(\phi_{\mathbf{s}}\right)=\bar{f}_{\mathbf{p}}^{0}$.

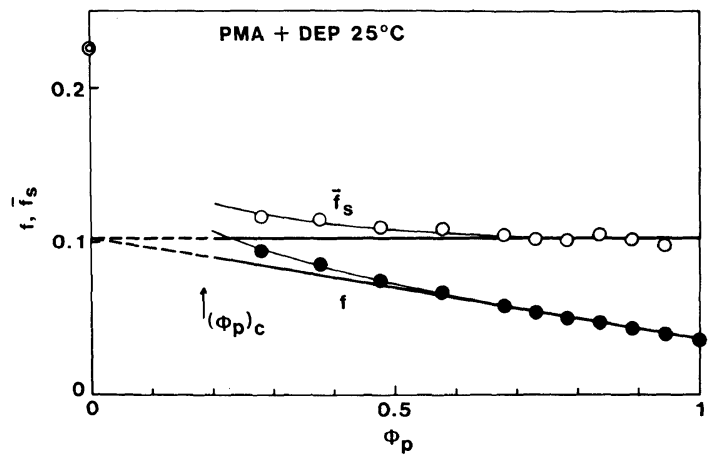

Figure 2. Composition dependence of $f\left(\phi_{\mathrm{s}}\right)(\boldsymbol{O})$ and $\bar{f}_{\mathrm{s}}$ $\left(\phi_{\mathrm{s}}\right)(\bigcirc)$ for poly(methylacrylate) in diethylphthalate at $25^{\circ} \mathrm{C}$. The unfilled circles were obtained in the way as mentioned in Figure 1.

equal to their limiting values at $\phi_{\mathrm{s}}=0$ not only in the vinicity of the undiluted polymer but over a fairly wide range of solvent volume fraction which probably covers sufficiently entangled solutions. In other words, the composition range in which the Fujita-Kishimoto approximation is valid is much wider than originally expected, and eq 45 would be a good approximation to describing $f$ in the entangled regime.

\section{Comments}

Since the mobility $m_{\mathrm{s}}$ is inversely proportional to the viscosity for pure liquids, eq 37 gives

$$
\ln \eta_{\mathrm{s}}^{0}=C_{\mathrm{s}}+B / \bar{f}_{\mathrm{s}}^{0}
$$

where the superscript 0 refers to the pure solvent. Therefore, if the temperature dependence of $\eta_{\mathrm{s}}^{0}$ obeys the WLF equation, $\bar{f}_{\mathrm{s}}^{0} / B$ can be determined. To obtain $\bar{f}_{\mathrm{s}}^{0}$ the parameter $B$ must be estimated. Since, as noted above, $B$ may be equated to $\xi$, we can resort to the method explained in describing the VrentasDuda theory. However, the value of $B$ so estimated cannot be used for eq 53 . The reason is the following.

At infinite dilution $\left(\phi_{\mathrm{s}} \rightarrow 1\right), m_{\mathrm{pj}}$ is inversely proportional to $\eta_{\mathrm{s}}^{0}$. This is the familiar Stokes law. Hence, $m_{\mathrm{pj}}(1)$ is directly proportional to $m_{\mathrm{s}}(1)$, which means that these two quantities have the same temperature dependence. Thus, it follows from eq 38 that $B$ (hence $\xi$ as well) has to approach unity as $\phi_{\mathrm{s}} \rightarrow 1$, so that, correctly, $\bar{f}_{\mathrm{s}}^{0}$ should be determined by eq 53 with $B=1$. The double circles on the ordinate axes in Figures 1 and 2 show the $\bar{f}_{\mathrm{s}}^{0}$ values computed on the basis of this idea. Both $f$ and $\bar{f}_{\mathrm{s}}$ in the figures should converge to these points as the polymer concentration is lowered.

The following remarks may be in order. First, $\bar{f}_{\mathrm{s}}^{*}$ is significantly lower than $\bar{f}_{\mathrm{s}}^{0}$. Second, the fact that $B=1$ at $\phi_{\mathrm{s}}=1$ implies that, in general, $B$ must the treated as compositiondependent. It would be inviting to reformulate a free theory of polymer solutions with this condition taken into account.

\section{REFERENCES}

1. H. Fujita, Fortsch. Hochpolym. Forsch., 3, 1 (1961)

2. J. S. Vrentas and J. L. Duda, J. Polym. Sci., Polym. Phys. Ed., 15, 403, 417 (1977); AIChE J., 25, 1 (1979).

3. C. W. Paul, J. Polym. Sci., Polym. Phys. Ed., 21, 425 (1983).

4. M. H. Cohen and D. Turnbull, J. Chem. Phys., 34, 120 (1961). 


\section{H. Fujita}

5. A. K. Doolittle, J. Appl. Phys., 22, 1471 (1951).

6. M. L. Willimas, R. F. Landel, and J. D. Ferry, $J$. Am. Chem. Soc., 77, 3701 (1955); J. D. Ferry, Viscoelastic Properties of Polymers, 3rd ed, John Wiley, New York, 1980, p 287.

7. R. N. Haward, J. Macromol Sci., Revs. Macromol. Chem., C4, 191 (1970).

8. H. Fujita and A. Kishimoto, J. Chem. Phys., 34, 393
(1961).

9. H. L. Frisch, D. Kempner, and T. K. Kwei, Macromolecules, 4, 237 (1971).

10. F. N. Kelley and F. Bueche, J. Chem. Phys., 50, 549 (1961).

11. R. D. Ferguson and E. von Meerwall, J. Polym. Sci. Polym. Phys. Ed., 18, 1285 (1980).

12. H. Fujita and Y. Einaga, Polymer, 31, 1486 (1990). 